\title{
Kinder rauchender Mütter sind asthmagefährdet
}

\author{
Eine Studie liefert Argumentationshilfen für Ärzte, die Schwangere vom Rauchen abhalten \\ wollen. Offenbar erhöht sich bereits in diesem Stadium das Asthmarisiko des Kindes.
}

_ Die Generation-R-Studie ist eine prospektive, bevölkerungsbasierte Kohortenstudie an schwangeren Frauen und ihren Kindern in Rotterdam. In jedem Trimester der Schwangerschaft werden eine körperliche Untersuchung und eine Ultraschalluntersuchung des Fötus durchgeführt. Die werdenden Mütter werden per Fragebogen unter Erfassung zahlreicher sozioökonomischer Parameter befragt. Insgesamt wurden über 6.000 Schwangerschaften zwischen 2002 und 2006 einbezogen.

Für die vorliegende Studie wurden die Vitalparameter des Kindes bei der Geburt, das Rauchverhalten der Mutter und des Vaters während der Schwangerschaft, die Exposition des Kindes gegenüber Zigarettenrauch sowie das Auftreten von Giemen und Asthma erfasst. Im Alter von sechs Jahren erfolgten eine Atemwiderstandsmessung mit der Unterbrechermethode (Rint) sowie die Bestimmung der Stickoxidfraktion im Exhalat (FeNO).

$25 \%(\mathrm{n}=1.503)$ der Mütter rauchten während der Schwangerschaft, davon $8,8 \%$ nur im ersten Trimester und 16,2\% während der gesamten Schwangerschaft. Von den Vätern rauchten $44 \%(\mathrm{n}=2.383)$ während der Schwangerschaft. 45,6\% der Kinder $(\mathrm{n}=2.149)$ hatten niemals Giemen, 28,6\% im frühen Kindesalter, $7,4 \%$ beim Schuleintritt und 18,5\% während der gesamten erfassten Studienperiode. Im Alter von sechs Jahren betrug der mittlere Atemwegswiderstand $0,84 \mathrm{kPa} / \mathrm{L} / \mathrm{s}$ und die mediane FeNO 7,5 ppb. Bei 4,8\% der Kinder $(\mathrm{n}=286)$ wurde vom Arzt ein Asthma bronchiale diagnostiziert.

Das fortgesetzte Rauchen der Mutter (mindestens fünf Zigaretten pro Tag) während der gesamten Schwangerschaft war mit einem erhöhten Risiko für frühes und anhaltendes Giemen (Odds Ratio [OR] 1,24) und Asthma (OR 1,65), nicht jedoch mit signifikanten Verände- rungen der Rint oder FeNO assoziiert. Diese Unterschiede konnten nicht durch Vitalparameter bei der Geburt erklärt werden. Rauchte die Mutter nur im ersten Trimenon, so hatte das keinen negativen Einfluss. Gleiches traf für das Rauchen des Vaters insgesamt zu.

- den Dekker HT, Sonnenschein-van der Voort AM, de Jongste $J C$ et al. Tobacco Smoke Exposure, Airway Resistance, and Asthma in School-age Children: The Generation R Study. Chest. 2015;148:607-17

\section{KOMMENTAR}

Nach diesen Ergebnissen ist ein intrauteriner Effekt des Nikotinkonsums wahrscheinlich. Werdende Mütter sollten das wissen. Ein anderer Schluss der Autoren erscheint fragwürdiger: Da das Rauchen des Vaters keinen Effekt zeigte, schließen sie aus, dass nicht erfasste sozioökonomische, verhaltensspezifische oder genetische Faktoren eine Rolle spielen. Eine kühne Schlussfolgerung, die zahlreichen anderen Studien widerspricht und vor allem den Umstand vernachlässigt, dass nur 70\% der Teilnehmer die Studie beendeten. Unter den Abbrechern waren überproportional häufig Kinder mit niedrigerem Geburtsgewicht, Eltern mit niedrigem Bildungsgrad, Raucher sowie Mütter mit hohem BMI und psychischen Belastungen während der Schwangerschaft. Das ist eine Fülle von Faktoren, die mit einem schlechteren
Start des Kindes ins Leben einhergehen. Auch die Befragung der Eltern bezüglich ihres Rauchverhaltens erscheint problematisch, $d a$ die Angaben möglicherweise geschönt sind. Einschlägige Biomarker wie Cotinin oder Nikotin in Urin, Blut oder Raumluft wurden in der Studie nicht bestimmt.

Prof. Dr. med.

H. S. FüeßI

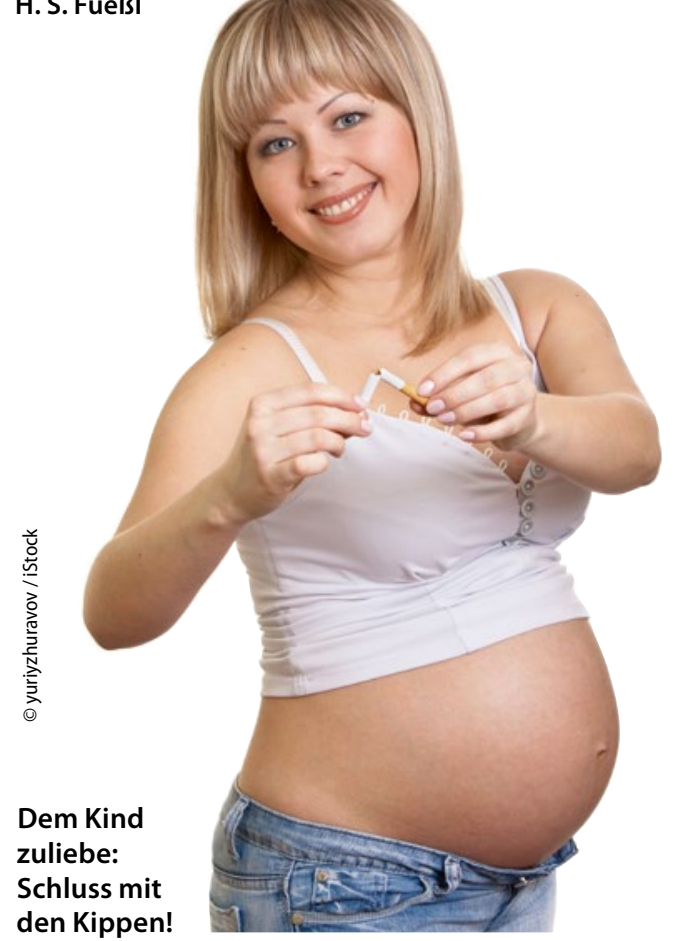

Hier steht eine Anzeige.

Springer 